\title{
Experimental Verification of CAPN1 and CAST Gene Polymorphisms in Different Generations of Da-Heng Broilers
}

\author{
Yu-Guang Zhou,, ${ }^{1}$ Yong Xiong,, Chao-Wu Yang, ${ }^{2}$ Xiao-Song Jiang, ${ }^{2}$ Jin-Shan Ran,, Jie Jin, \\ Ye Wang, ${ }^{1}$ Dan Lan, ${ }^{1}$ Peng Ren, ${ }^{1}$ Yao-Dong Hu, ${ }^{1}$ and Yi-Ping Liu ${ }^{1}$ \\ ${ }^{1}$ Farm Animal Genetic Resources Exploration and Innovation Key Laboratory of Sichuan Province, Sichuan Agricultural University, \\ Chengdu Campus, Chengdu 611130, China \\ ${ }^{2}$ Sichuan Animal Science Academy, Chengdu 610066, China \\ Correspondence should be addressed to Yi-Ping Liu; liuyp578@yahoo.com
}

Received 27 October 2016; Revised 9 March 2017; Accepted 2 May 2017; Published 21 June 2017

Academic Editor: Leon Spicer

Copyright (c) 2017 Yu-Guang Zhou et al. This is an open access article distributed under the Creative Commons Attribution License, which permits unrestricted use, distribution, and reproduction in any medium, provided the original work is properly cited.

\begin{abstract}
The micromolar calcium activated neutral protease (CAPN1) and calpastatin (CAST) have been widely regarded as genes related to muscle growth and meat tenderness. The objective of this study was to verify the association of SNPs of CAPN1 and CAST genes with carcass and tenderness traits and search the possible change patterns of SNPs in CAPN1 and CAST genes in six generations of broiler breeding process for growth rate, efficiency, and reproduction, during the third generation and the ninth generation, respectively. We found that, for CAPN1, genetic effects between SNPs (G3535A, C7198A) and meat tenderness were similar in different generations, while SNP3 (G7324A) was a novel polymorphism and had significant association with carcass and tenderness traits $(P<0.05)$ in this study. Furthermore, there was significant association between SNP4 (G9950A) and carcass indexes instead of tenderness traits $(P<0.05)$ which was consistent in the two generations. Moreover, although SNP6 (G37868A) of CAST had no relevance to carcass traits or tenderness traits in the third generation, it showed significant association with LW and CW in the ninth generation $(P<0.05)$.
\end{abstract}

\section{Introduction}

Carcass traits can intuitively reflect the production of broilers. Color, drip loss, juiciness, tenderness, and flavor are the major traits for chickens, among which tenderness can directly affect the taste of chicken [1]. However, tenderness is a complex trait in breeding programs for its complex indexes and factors [2]. With the development of molecular technology, molecular markers have a good theoretical basis in correlating with production traits and improve the breeding process [3].

In the past two decades, genetic markers related to production traits and tenderness traits have been largely reported [4-6]. Among these genes, calpain systen has been widely reported to be related to postmortem muscle proteolysis and tenderization $[7,8]$. Calpain is a ubiquitous cytoplasmic cysteine protease requiring calcium ions for activity [9]. Calpain system consists of the ubiquitously expressed $\mu$-calpain (CAPN1) and m-calpain (CAPN2) and the only endogenous inhibitor of CAPN1 and CAPN2-calpastatin (CAST) [10]. Excitation of CAPN1 needs calcium at micromolar concentrations and CAPN2 requires calcium for activity at millimolar concentrations [11]. CAPN1 gene encodes the large subunit of $\mu$-calpain and the CAST gene encodes inhibitor of the calpains [12]. CAPN1 gene is located in chicken chromosome 3 with 14200 bp in length and contains 21 exons and 20 introns, while CAST gene consisting of 31 exons and 30 introns is mapped to chromosome $\mathrm{Z}$, with $61188 \mathrm{bp}$ in length.

Currently, multiple polymorphisms of genes in calpain system have been identified as potentially relevant to meat quality traits. Of these, CAPN1 has a significant association with postmortem proteolysis and meat tenderization $[13,14]$, 
while CAPN2 was thought to play a marginal role [15]. Two nonsynonymous single nucleotide polymorphisms (SNPs) of CAPN1 gene, C316G and A530G, have been found to be associated with meat quality traits in cattle [16-19]. At the same time C4751T in CAPN1 has a significant association with shear force at 7, 14, and 21 days of postmortem [20]. In chickens, four SNPs (C2546T, G3535A, C7198A, and G9950A) of CAPN1 gene are associated with meat quality and tenderness traits according to Shu et al's and Felício et al.s researches $[21,22]$. Simultaneously, several polymorphisms of CAST have also been described and identified to be associated with carcass quality and production traits [23, 24]. Among these markers, a SNP in the intron 5 of the bovine CAST gene, AY_008267.1:g282CNG, is associated with postmortem meat tenderness in crossbred B. taurus populations [25]. In 2010, Liu et al. found that a polymorphism (C36127T) of CAST gene has relation with carcass and tenderness traits in chickens [26]. Recently, Biswas et al. conducted an experiment to detect the change of expression of CAST in muscle of postmortem aging of meat during holding at refrigeration temperatures with the real-time PCR and found association between CAST gene and muscle change in chickens [27].

In our previous researches, we conducted association study of CAPN1 and CAST with carcass and tenderness traits in the third generation of Da-Heng broilers. The results indicate that several SNPs in the exons or introns have significant effects on chicken carcass and tenderness traits $[28,29]$. However, limited information about the change and clarification of SNPs and haplotypes after several generations is available. Is there any tendency or pattern? Therefore, to verify our hypothesis, we studied the association of CAPN1 and CAST gene with carcass and tenderness traits in the ninth generation of $\mathrm{Da}-\mathrm{Heng}$ broiler and conducted a comparison analysis between our previous studies in the third generation.

\section{Materials and Methods}

2.1. Chicken Population. The ninth generation of Da-Heng high-quality broilers, including five pure lines S01, S05, S06, S07, and S08 and the crossbred S01 $\times$ S08 line, were used in this study. Da-Heng high-quality broiler is a specialized meat type breeding with native chickens in Sichuan and Guangdong provinces of China by Sichuan Da-Heng Poultry Breeding Company. The selection focus of Da-Heng broiler breeding lines is different, such as growth rate and feed conversion rate. But the breeding of all the lines takes into account the carcass traits, and some takes into account the meat quality. Two-line crossbreeding S01 $\times$ S08 is a commercial crossbreed. Except for S01, each strain was randomly sampled with 30 male chickens and 30 female chickens for slaughtering and blood collecting. For S01, 35 male chickens and 35 female chickens were randomly sampled. Briefly, a total of $370 \mathrm{Da}-H e n g$ broilers of the ninth generation were sampled for sequencing.

2.2. Management and Slaughter Parameter Measurements. All chickens were fed based on the National Research Council's (NRC from 2014) requirements of broilers. At the age of 90 days, chickens were slaughtered by electric shock and blood samples were collected. Slaughter parameters including live weight (LW), carcass weight (CW), leg muscle weight (LMW), abdominal fat weight (AFW), skin fat thickness (SFT), breast muscle weight (BMW), semieviscerated weight (SEW), and eviscerated weight (EW) were measured according to the following description. The CW was measured on the chilled carcass after removal of feathers. SEW is a parameter of carcass weight of removal of the trachea, oesophagus, gastrointestinal tract, spleen, pancreas, and gonad. EW is a parameter of carcass weight of removal of all internal organs which was measured on the SEW after removal of the head, claws, heart, liver, gizzard, glandular stomach, and abdominal fat. The ratios of these traits to $\mathrm{CW}$ were calculated as eviscerated percentage (EP), semieviscerated percentage (SEP), breast muscle percentage (BMP), leg muscle percentage (LMP), and abdominal fat percentage (AFP). Tenderness was measured by Warner-Bratzler shear force (SF), which determines the relative force required to pass a blunt blade through a section of meat [30]. Water depletion rate (WDR) is drip losses rate measured according to the American Meat Science Association (AMSA) (1995) guidelines. All protocols used in this study were approved by Sichuan Agricultural University Animal Care and Use Committees.

2.3. DNA Extraction and PCR Amplification. Genomic DNA was extracted from the blood samples $(15 \mu \mathrm{l})$ by phenolicchloroform method after digestion with proteinase $\mathrm{K}$ and precipitation with $\mathrm{NaCl}$ and alcohol.

The primer pairs CAST_SNP and CAPN1_SNP (Table 1) were designed by Primer Premier 5.0 according to the GenBank accession numbers NC_006127.4 and NT_464263.1, respectively. Cycling conditions consisted of an initial denaturation at $94^{\circ} \mathrm{C}$ for $6 \mathrm{~min}$ and 35 cycles at $94^{\circ} \mathrm{C}$ for $45 \mathrm{~s}$, $48^{\circ} \mathrm{C}$ or $56^{\circ} \mathrm{C}$ (for CAST and CAPN1, resp.) for $45 \mathrm{~s}$, and $72^{\circ} \mathrm{C}$ for $1 \mathrm{~min}$, followed by a final step of $72^{\circ} \mathrm{C}$ for $8 \mathrm{~min}$. PCR amplifications were performed in a total volume of $25 \mu \mathrm{l}$ containing $2.0 \mu \mathrm{l}(50 \mathrm{ng} / \mu \mathrm{l})$ of template, $12.5 \mu \mathrm{l}$ of $2 \mathrm{x}$ Taq PCR MasterMix, $8.5 \mu \mathrm{l}$ of $\mathrm{ddH}_{2} \mathrm{O}$, and $1.0 \mu \mathrm{l}$ of each primer $(10 \mathrm{pmol} / \mu \mathrm{l})$. PCR amplification product was detected by $1 \%$ agarose gel and gels were both visualized on Gel DocTMEQ170-8060 and photographed under UV light.

The PCR products were sequenced by TSINGKE Biological Technology Corporation, Sichuan, China.

2.4. Statistical Analysis. Data were analyzed by General Linear Model (GLM) procedures of SAS (SAS Inst. Inc., Cary, NC, USA). The line and genetic effects were analyzed by the following model: $Y=\mu+L+S+G+(L \times G)+(S \times G)+e$, where $Y$ is the traits measured on chickens, $\mu$ is the population mean, $e$ is the random error, $L$ is the fixed effect of line, $S$ is the fixed effect of sex, $G$ is the fixed effect associated with the genotype, $(L \times G)$ is the interaction between the line and genotype, and $(S \times G)$ is the interaction between the sex and genotype and it was excluded from the model if its value was $P>0.05$ on a given trait. The values were presented as least square means \pm standard error. The significance of least square means was tested with the Duncan test $(P<0.01$ or $P<0.05)$. 
TABLE 1: Primers for screening in the chicken CAPN1 and CAST genes.

\begin{tabular}{|c|c|c|c|c|c|}
\hline & Primers & Sequence of the primers & Amplified length & Binding regions & $\operatorname{Tm}\left({ }^{\circ} \mathrm{C}\right)$ \\
\hline \multirow{8}{*}{ CAPN1 } & \multirow{2}{*}{$\mathrm{P} 1$} & F: TCA CCT CAC GTG CCT CTC TCA & \multirow{2}{*}{217} & \multirow{2}{*}{ Exon 5} & \multirow{2}{*}{58.0} \\
\hline & & R: CGG AAC ACT TAC GTC GAT & & & \\
\hline & \multirow{2}{*}{$\mathrm{P} 2$} & F: AGG GGT AGG GTA ATA GAA CTA & \multirow{2}{*}{233} & \multirow{2}{*}{ Exon 6} & \multirow{2}{*}{58.0} \\
\hline & & R: ACC GCC AGC CAT CAA AT & & & \\
\hline & \multirow{2}{*}{ P3 } & F: CCT CCT TCC TCC TCA GAC AAA & \multirow{2}{*}{191} & \multirow{2}{*}{ Exon 16} & \multirow{2}{*}{55.0} \\
\hline & & R: CAGCCT TGG CAC AAC TAG AGA & & & \\
\hline & \multirow{2}{*}{$\mathrm{P} 4$} & F: TCAGGACACTGG TGT TCA ATA & \multirow{2}{*}{212} & \multirow{2}{*}{ Intron 3} & \multirow{2}{*}{55.0} \\
\hline & & R: GGA AAG GGT GTA GTG GTA C & & & \\
\hline \multirow{6}{*}{ CAST } & \multirow{2}{*}{ P5 } & F: AAT ACA GGG TCA CAT CG & \multirow{2}{*}{239} & \multirow{2}{*}{ Exon 8} & \multirow{2}{*}{56.0} \\
\hline & & R: AAA GAA ACA TTC CCT GA & & & \\
\hline & \multirow{2}{*}{ P6 } & F: AAA CGA GAA GGT AGC C & \multirow{2}{*}{291} & \multirow{2}{*}{ Exon 11} & \multirow{2}{*}{55.0} \\
\hline & & R: CTG GTA TCT TTG GAA GAC ATA & & & \\
\hline & \multirow{2}{*}{ P7 } & F: CCA AAA GTA GAT GAA CAT TCT & \multirow{2}{*}{249} & \multirow{2}{*}{ Intron 11} & \multirow{2}{*}{48.0} \\
\hline & & R: GCT TCT ATT AAT TCC TAC CT & & & \\
\hline
\end{tabular}

\section{Results}

3.1. Detection of Polymorphisms in CAPN1 and CAST Genes in the Ninth Generation. In this study (Table 3), a total of four polymorphisms were detected in CAPN1 gene, including a G3535A (NC_006090.1:g.30419210G $<$ A) transition in exon 6, a C7198A (NC_006090.1:g.30422873C<A) transition, a G7324A (NC_006090.1:g.30422999G<A) transition in exon 16, and a G9950A (NC_006090.1:g.30425625G<A) transition in intron 13. As for CAST gene, only a G37868A (NC_006127.2:g.57042952G<A) transition was found in intron 11.

Hardy-Weinberg equilibrium test of the 5 SNPs was shown in Table 3. As expected, all of the SNPs fitted the assumption of the Hardy-Weinberg equilibrium $(P>0.05)$ and the minor allele frequencies of all the mutations were more than 0.01 . These results indicated that the observed heterozygosities of all SNPs in the ninth generations were at similar levels.

3.2. Genotype and Allele Frequencies in the Ninth Generations. The allele and genotype frequencies of the CAPN1 and CAST obtained in different lines of the ninth generation were shown in Table 5. Chi-squared test suggested that SNP1 and SNP2 had significant correlations within each line, while SNP3 and SNP4 were not. For SNP1, SNP3, and SNP4, GG genotype was the favorable homozygote in most lines, and $\mathrm{G}$ was the advantageous allele. For SNP2, the frequency of allele $C$ was higher than allele $\mathrm{A}$, and $\mathrm{CC}$ genotype was the favorable one followed by AC and AA genotype. As for the SNP6 of CAST, genotype GG was rare and A is the advantageous allele.

3.3. Genetic Effect of CAPN1 and CAST Gene on Carcass and Tenderness Traits. The results of association analysis in the ninth generation were summarized in Table 7 . Our results showed that, in the ninth generation, SNP1 was significantly associated with BMW, SFT, SF $(P<0.05)$, and FT $(P<0.01)$ and indexes of genotype AG and GG were significantly higher than AA. SNP2 had a significant correlation with SEP and FT $(P<0.05)$ and AFW and AFP $(P<0.01)$, while genotype CC was significantly higher than the other two genotypes. SNP3 had a significant relation with LW, SEP, and SF $(P<0.05)$ and highly significant association with $\mathrm{CW}(P<0.01)$; genotypes AA and AG were predominant for LW and CW. A significant association between SNP4 and LW, CW, BMW, and LMW $(P<0.05)$ was also observed, and indexes of homozygous GG were significantly higher. In CAST, there was a significant association between SNP5 and LW and CW $(P<0.05)$.

\section{Discussion}

Both the carcass and tenderness traits are controlled by multiple genes [30]. Thus, understanding their genetic basis will promote the genetic improvement of the carcass and tenderness traits. The association analysis of candidate gene is one of the main methods to determine whether specific genes are associated with specific traits in economic animals.

Previous studies found that calpain system can improve muscle tenderness after slaughter by cleaved limited myofibrillar proteins such as titin, desmin, and vinculin, while high levels of CAST are related to decrease of proteolysis and increase of meat toughness [31]. Thus, CAPN1 and CAST probably have significant effects on meat tenderness. In fact, the genotypic effects of CAPN1 are able to significantly affect the carcass traits in many species [29, 32, 33]. However, reports about the effects of CAST on carcass traits instead of tenderness traits are rare in chickens.

The Hardy-Weinberg equilibrium is influenced by many factors, including selection, the rate of recombination, the rate of mutation, genetic drift, the system of mating, population structure, and genetic linkage. As expected, due to lack of foreign blood in current chicken population, the observed heterozygosity of all SNPs was at a similar level, and all SNPs fit the assumption of the Hardy-Weinberg equilibrium. 
TABLE 2: The Hardy-Weinberg equilibriumof CAPN1 and CAST gene mutation in the third generation.

\begin{tabular}{lccccccc}
\hline Markers & Position & ObsHET & ExptHET & Allele change & Amino acids change & HWE $(P)$ & MAF \\
\hline SNP1 & 3535 & 0.360 & 0.328 & G $>$ A & 215 Leu > Leu & 0.094 & 0.371 \\
SNP2 & 7198 & 0.462 & 0.370 & C $>$ A & 427 Glu > His & 0.185 \\
SNP4 & 9950 & 0.637 & 0.396 & G $>$ A & 932 Cys > Arg & 0.683 \\
SNP5 & 37752 & 0.368 & 0.853 & A $>$ T & 307 Lys $>$ Lys & 0.751 & 0.339 \\
SNP6 & 37868 & 0.621 & 0.376 & G $>$ A & 335 Arg $>$ His & 0.964 & 0.454 \\
\hline
\end{tabular}

Notes. ObsHET, observed heterozygosity; ExptHET, expected heterozygosity; HWE, Hardy-Weinberg equilibrium; MAF, minimum allele frequency.

TABLE 3: The Hardy-Weinberg equilibriumof CAPN1 and CAST gene mutation in the ninth generation.

\begin{tabular}{lccccccc}
\hline Markers & Position & ObsHET & ExptHET & Allele change & Amino acids change & HWE $(P)$ & MAF \\
\hline SNP1 & 3535 & 0.439 & 0.5 & G $>$ A & 215 Leu > Leu & 0.150 & 0.488 \\
SNP2 & 7198 & 0.396 & 0.44 & C $>$ A & 427 Glu > His & 0.262 \\
SNP3 & 7324 & 0.488 & 0.473 & G $>$ A & 489 Val > Val & 0.326 \\
SNP4 & 9950 & 0.53 & 0.496 & G $>$ A & 932 Cys > Arg & 0.350 \\
SNP6 & 37868 & 0.488 & 0.498 & G $>$ A & 335 Arg $>$ His & 0.384 \\
\hline
\end{tabular}

Notes. ObsHET, observed heterozygosity; ExptHET, expected heterozygosity; HWE, Hardy-Weinberg equilibrium; MAF, minimum allele frequency.

Therefore, the five SNPs in the ninth generation are suitable for the association study.

Numerous studies report that calpain system plays important roles in carcass and tenderness traits [21, 34, 35]. In the present study, we examined CAPN1 and CAST as two candidate genes for carcass and tenderness traits in the ninth generation of a commercial chicken breed and detected four SNPs in CAPN1 and one SNP in CAST (Table 3). Compared with our previous research (Table 2), SNP3 in CAPN1 was a novel mutation and had never been reported in previous studies. Our results suggested that SNP3 has a significant association with LW, CW, SEP, and SF. This may be due to genetic variation benefitting from meat quality breeding that resulted from specific environment and artificial selection during the breeding process. This mutation created a synonymous transition and may influence the transcription or translation of CAPN1 gene. We did not detect A37752T in CAST in the ninth generation probably because of stronger artificial selection. In our previous study, there are significant associations between SNP5 and LMW $(P<0.05)$, CW, LW, and BMW $(P<0.01)$ in the third generation (Table 6). And we found individuals of genotype AA in SNP5 had significantly higher indexes than individuals of genotypes AT and TT. After intense artificial selection we reserved individuals of genotype AA and wiped out genotype AT and TT. This could be the reason of the miss of SNP5 in the ninth generation.

In order to investigate the genetic changes of $C A P N 1$ and $C A S T$ during the breeding process, we analyzed frequencies of alleles and genotypes of CAPN1 and CAST in the third and the ninth generation. We found that all SNPs of CAPN1 in the third generation were significantly associated within lines, while SNP4 and new site, SNP3, were not correlated with lines in the ninth generation. This indicated that difference of SNP4 in each line tended to reduce because of the lack of foreign blood. We found that the frequency of allele A in SNP2 of the ninth generation was higher than the third generation. It is interesting that the frequencies of allele in SNP6 in the two generations were extremely different. In the third generation, frequency of allele $G$ was 0.540 , slightly higher than allele A (Table 4). However, frequency of allele $\mathrm{A}$ in the ninth generation was 0.719 , obviously higher than allele $\mathrm{G}$. This situation could be explained by the combination of genetic effects of two generations.

In the breeding process of Da-Heng broilers, we found that carcass indexes of the ninth generation were obviously higher than the third generation. In the third generation of Da-Heng broilers, we found that SNP1 had significant effect on BMP and SF and individuals of genotype GG had higher carcass indexes and better tenderness indexes, while, in ninth generation, SNP1 had significant association with BMW, SFT, SF, and WDR, and GG genotype still had higher carcass indexes and better tenderness traits. SNP2 in the third generation showed significant association with AFW and WDR. And there was significant correlation between SNP2 and SEP, AFW, AFP, and WDR in the ninth generation, and chickens of genotype CC had a higher AFW. The loss of genotype $\mathrm{CC}$ during the breeding process is probably because we need individuals with lower AFW. As a result, frequency of allele $\mathrm{C}$ in the ninth generation was lower than the third generation. SNP4 in the ninth generation had significant association with CW, LW, BMW, and LMW, and individuals of genotype GG had higher carcass indexes. This was similar to the third generation. These sites were relatively conservative during the breeding process. In the third generation, there was no association between SNP6 in CAST and carcass or tenderness traits, while SNP6 showed significant association with LW and CW in the ninth generation. Collectively, the association of SNP1 and SNP2 as well as SNP4 between carcass and tenderness traits was consistent in the two generations.

We found that carcass indexes and tenderness indexes in the ninth generation were higher than those in the third generation (Tables 6 and 7). And furthermore, individuals 
TABLE 4: Genotype frequency and allele frequency of CAPN1 and CAST gene in the third generation.

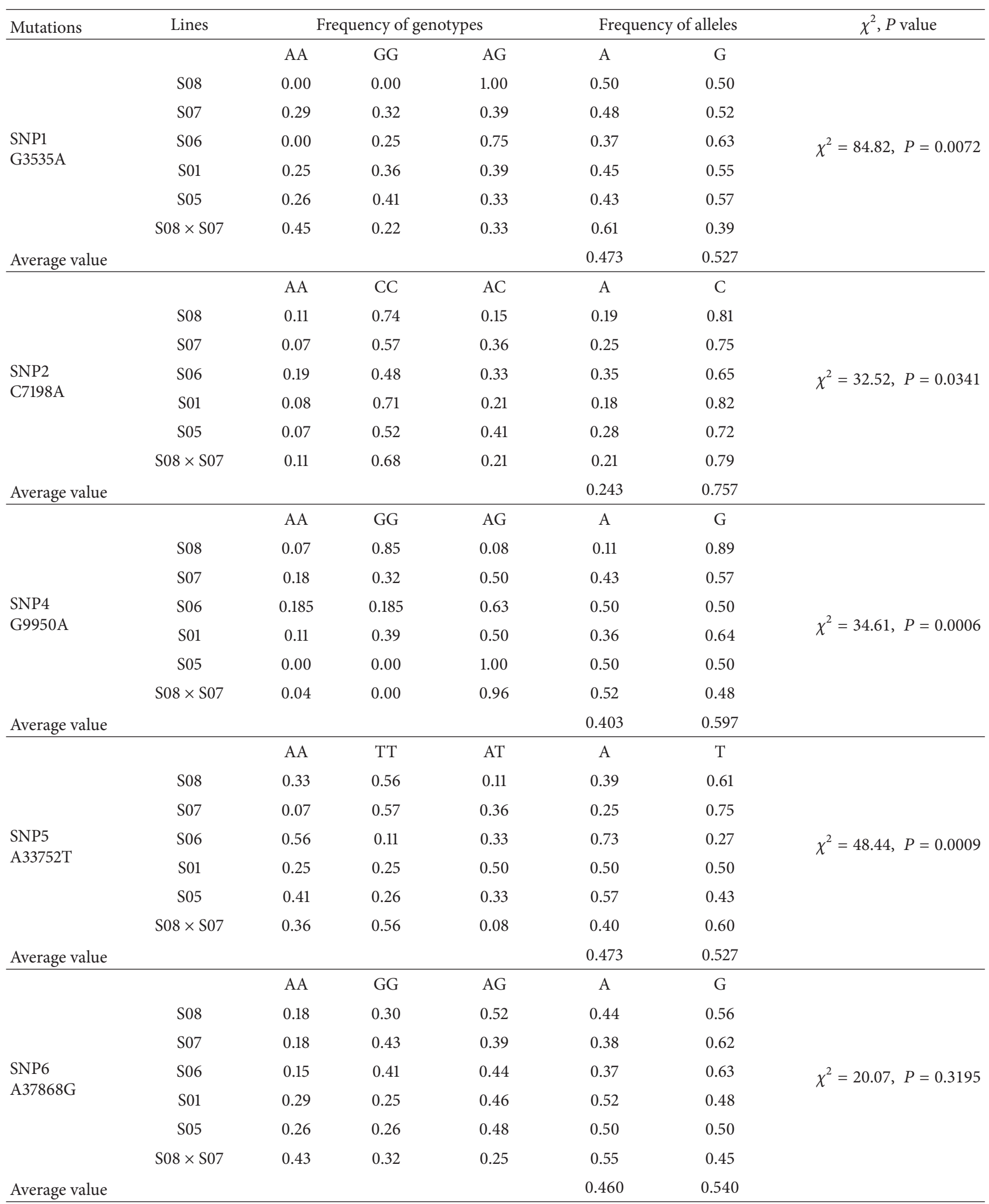


TABLE 5: Genotype frequency and allele frequency of CAPN1 and CAST gene in the ninth generation.

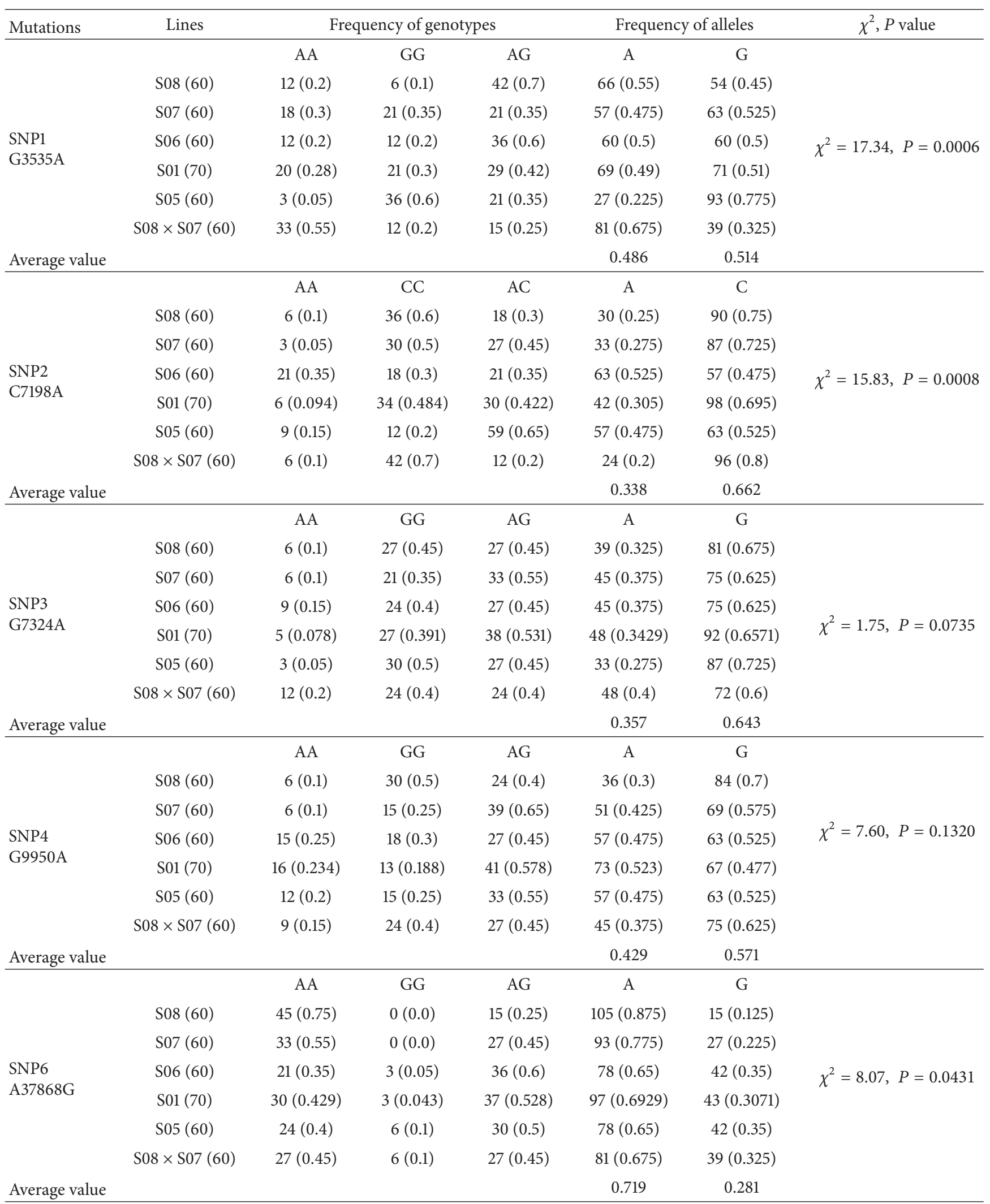




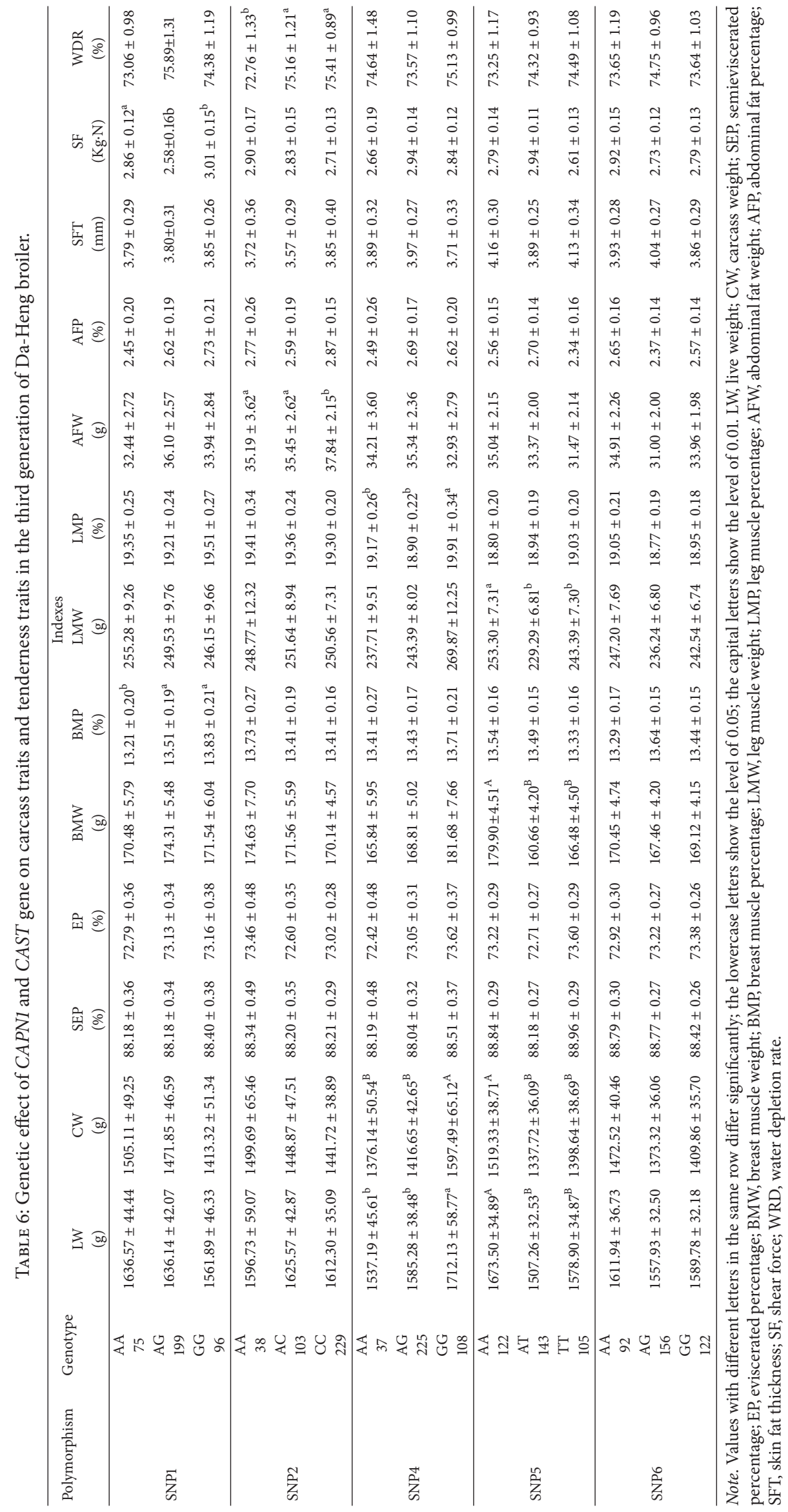




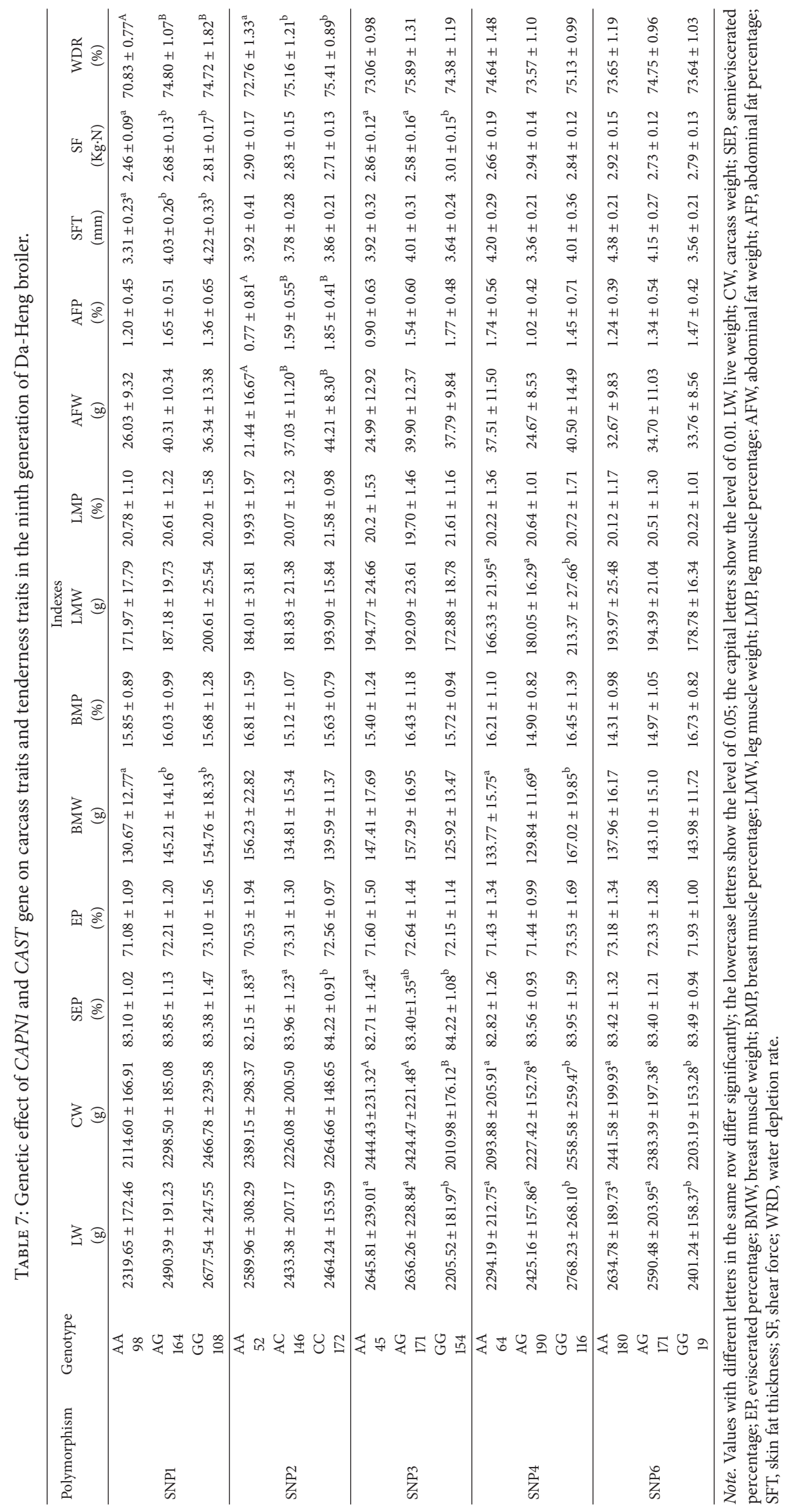


with better carcass and tenderness indexes have higher related genotype frequency. That is to say, the change of SNPs in these two genes has a direction of clarification under our breeding progress for meat quality. However, we did not conduct the comparison analysis with the original generation; this limited us from getting more information about the dynamic tendency of SNPs and haplotypes.

\section{Conclusion}

In conclusion, the association between SNP1, SNP2, and SNP4 in CAPN1 gene with carcass and tenderness traits of different generations was very consistent. And these sites have slight changes toward the direction of benefitting meat type breeding under the pressure of artificial selection of broilers. We can use these consistent molecular markers for genetic markers assistant selection in chickens. We can also regard SNP3 of CAPN1 as a potential molecular marker related to carcass and tenderness traits. In CAST, SNP5 might be a molecular marker for molecular assisted selection. However, the association between SNP6 and carcass as well as tenderness traits needs further verification. And the change of SNPs in the two genes has a direction of clarification during the six generations of breeding.

\section{Conflicts of Interest}

All authors have not declared any conflicts of interest.

\section{Acknowledgments}

This research was supported by the Open Fund of Farm Animal Genetic Resources Exploration and Innovation Key Laboratory of Sichuan Province (Grant no. 2016NYZ0043).

\section{References}

[1] R. A. Mancini and M. C. Hunt, "Current research in meat color," Meat Science, vol. 71, no. 1, pp. 100-121, 2005.

[2] L. F. Pinto, J. B. Ferraz, F. V. Meirelles et al., "Association of SNPs on CAPN1 and CAST genes with tenderness in Nellore cattle," Genetics \& Molecular Research, vol. 9, no. 3, pp. 1431-1342, 2010.

[3] W. Reardon, A. M. Mullen, T. Sweeney, and R. M. Hamill, "Association of polymorphisms in candidate genes with colour, water-holding capacity, and composition traits in bovine M. longissimus and M. semimembranosus," Meat Science, vol. 86, no. 2, pp. 270-275, 2010.

[4] M. L. Wolcott and D. J. Johnston, "The impact of genetic markers for tenderness on steer carcass and feedlot exit and heifer puberty traits in Brahman cattle," in Proceedings of Matching Genetics \& Environment: A New Look at An Old Topic Conference of the Association for the Advancement of Animal Breeding \& Genetics, 2009.

[5] M. M. Cabling, H. S. Kang, B. M. Lopez et al., "Estimation of genetic associations between production and meat quality traits in Duroc pigs," Asian-Australasian Journal of Animal Sciences, vol. 28, no. 8, pp. 1061-1065, 2015.

[6] L. Di Stasio, A. Brugiapaglia, G. Destefanis et al., "GH1 as candidate gene for variability of meat production traits in
Piemontese cattle," Journal of Animal Breeding and Genetics, vol. 120 , no. 5, pp. 358-361, 2003.

[7] W. Barendse, B. E. Harrison, R. J. Bunch, and M. B. Thomas, "Variation at the Calpain 3 gene is associated with meat tenderness in zebu and composite breeds of cattle," BMC Genetics, vol. 9, article no. 41, 2008.

[8] J. Xin, Z. Li-chun, L. Zhao-Zhi, L. Xiao-hui, J. Hai-Guo, and Y. Chang-Guo, "Association of polymorphisms in the calpain I gene with meat quality traits in yanbian yellow cattle of China," Asian-Australasian Journal of Animal Sciences, vol. 9 article 41, pp. 9-16, 2011.

[9] H. Sorimachi, A. Freiburg, B. Kolmerer et al., "Tissue-specific expression and $\alpha$-actinin binding properties of the Z-disc titin: implications for the nature of vertebrate Z-discs," Journal of Molecular Biology, vol. 270, no. 5, pp. 688-695, 1997.

[10] D. E. Goll, V. F. Thompson, H. Q. Li, W. Wei, and J. Y. Cong, "The calpain system," Physiological Reviews, vol. 83, no. 3, pp. 731-801, 2003.

[11] K. Suzuki and H. Sorimachi, "A novel aspect of calpain activation," FEBS Letters, vol. 433, no. 1-2, pp. 1-4, 1998.

[12] G. Gandolfi, L. Pomponio, P. Ertbjerg et al., "Investigation on CAST, CAPN1 and CAPN3 porcine gene polymorphisms and expression in relation to post-mortem calpain activity in muscle and meat quality," Meat Science, vol. 88, no. 4, pp. 694-700, 2011.

[13] G. H. Geesink, S. Kuchay, A. H. Chishti, and M. Koohmaraie, " $\mu$-calpain is essential for postmortem proteolysis of muscle proteins," Journal of Animal Science, vol. 84, no. 10, pp. 28342840, 2006.

[14] C. M. Kemp, P. L. Sensky, R. G. Bardsley, P. J. Buttery, and T. Parr, "Tenderness-an enzymatic view," Meat Science, vol. 84, no. 2, pp. 248-256, 2010.

[15] J. P. Camou, J. A. Marchello, V. F. Thompson, S. W. Mares, and D. E. Goll, "Effect of postmortem storage on activity of $\mu$ - and mcalpain in five bovine muscles," Journal of Animal Science, vol. 85, no. 10, pp. 2670-2681, 2007.

[16] H. S. Cheong, D.-H. Yoon, B. L. Park et al., "A single nucleotide polymorphism in CAPN1 associated with marbling score in Korean cattle," BMC Genetics, vol. 9, article 33, 2008.

[17] P. Corva, L. Soria, A. Schor et al., "Association of CAPN1 and CAST gene polymorphisms with meat tenderness in Bos taurus beef cattle from Argentina," Genetics and Molecular Biology, vol. 30, no. 4, pp. 1064-1069, 2007.

[18] B. T. Page, E. Casas, M. P. Heaton et al., "Evaluation of singlenucleotide polymorphisms in CAPN1 for association with meat tenderness in cattle," Journal of Animal Science, vol. 80, no. 12, pp. 3077-3085, 2002.

[19] B. T. Page, E. Casas, R. L. Quaas et al., "Association of markers in the bovine CAPN1 gene with meat tenderness in large crossbred populations that sample influential industry sires," Journal of Animal Science, vol. 82, no. 12, pp. 3474-3481, 2004.

[20] S. N. White, E. Casas, T. L. Wheeler et al., "A new single nucleotide polymorphism in CAPN1 extends the current tenderness marker test to include cattle of Bos indicus, Bos taurus, and crossbred descent," Journal of Animal Science, vol. 83, no. 9, pp. 2001-2008, 2005.

[21] J. T. Shu, M. Zhang, Y. J. Shan et al., "Analysis of the genetic effects of CAPN1 gene polymorphisms on chicken meat tenderness," Genetics and Molecular Research, vol. 14, no. 1, pp. 13931403, 2015.

[22] A. M. Felício, C. Boschiero, J. C. Balieiro et al., "Identification and association of polymorphisms in CAPN1 and CAPN3 
candidate genes related to performance and meat quality traits in chickens," Genetics and Molecular Research, vol. 12, no. 1, pp. 472-482, 2013.

[23] A. K. Lindholm-Perry, G. A. Rohrer, J. W. Holl et al., "Relationships among calpastatin single nucleotide polymorphisms, calpastatin expression and tenderness in pork longissimus," Animal Genetics, vol. 40, no. 5, pp. 713-721, 2009.

[24] M. Škrlep, M. Čandek-Potokar, T. Kavar et al., "Association of PRKAG3 and CAST genetic polymorphisms with traits of interest in dry-cured ham production: comparative study in France, Slovenia and Spain," Livestock Science, vol. 128, no. 1-3, pp. 60-66, 2010.

[25] F. S. Schenkel, S. P. Miller, Z. Jiang et al., "Association of a single nucleotide polymorphism in the calpastatin gene with carcass and meat quality traits of beef cattle," Journal of Animal Science, vol. 84, no. 2, pp. 291-299, 2006.

[26] A. F. Liu, "Single nucleotide polymorphism of calpastatin gene and correlation analysis with meat traits in chicken," Chinese Journal of Veterinary Science, vol. 30, no. 12, pp. 1693-1694, 2010.

[27] A. K. Biswas, S. Tandon, and C. K. Beura, "Identification of different domains of calpain and calpastatin from chicken blood and their role in post-mortem aging of meat during holding at refrigeration temperatures," Food Chemistry, vol. 200, pp. 315321, 2016.

[28] H.-H. Chai, D. Lim, E. Jung, B.-H. Choi, and Y.-M. Cho, "Understanding the interaction determinants of CAPN1 inhibition by CAST4 from bovines using molecular modeling techniques," Molecules, vol. 19, no. 9, pp. 14316-14351, 2014.

[29] Z.-R. ZHANG, Q. ZHU, and Y.-P. LIU, "Correlation analysis on single nucleotide polymorphism of CAPN1 gene and meat quality and carcass traits in chickens," Agricultural Sciences in China, vol. 6, no. 6, pp. 749-754, 2007.

[30] Z. R. Zhang, Y. P. Liu, X. Jiang, H. R. Du, and Q. Zhu, "Study on association of single nucleotide polymorphism of CAPN1 gene with muscle fibre and carcass traits in quality chicken populations," Journal of Animal Breeding and Genetics, vol. 125, no. 4, pp. 258-264, 2008.

[31] K. L. Voges, C. L. Mason, J. C. Brooks et al., "National beef tenderness survey-2006: assessment of Warner-Bratzler shear and sensory panel ratings for beef from US retail and foodservice establishments," Meat Science, vol. 77, no. 3, pp. 357364, 2007.

[32] Y. Maeda, K. Kawabe, S. Okamoto et al., "Genetical studies on muscle protein turnover rate and calcium activated neutral protease activity in the skeletal muscle of the Japanese quail, coturnix coturnix japonica," Nihon Chikusan Gakkaiho, vol. 62, no. 9, pp. 813-821, 1991.

[33] C. A. Morris, N. G. Cullen, S. M. Hickey et al., "Genotypic effects of calpain 1 and calpastatin on the tenderness of cooked M. longissimus dorsi steaks from Jersey x Limousin, Angus and Hereford-cross cattle," Animal Genetics, vol. 37, no. 4, pp. 411414, 2006.

[34] R. A. Curi, L. A. L. Chardulo, J. Giusti, A. C. Silveira, C. L. Martins, and H. N. de Oliveira, "Assessment of GH1, CAPN1 and CAST polymorphisms as markers of carcass and meat traits in Bos indicus and Bos taurus-Bos indicus cross beef cattle," Meat Science, vol. 86, no. 4, pp. 915-920, 2010.

[35] T. Smith, M. G. Thomas, T. D. Bidner et al., "Single nucleotide polymorphisms in Brahman steers and their association with carcass and tenderness traits," Genetics and Molecular Research, vol. 8, no. 1, pp. 39-46, 2009. 

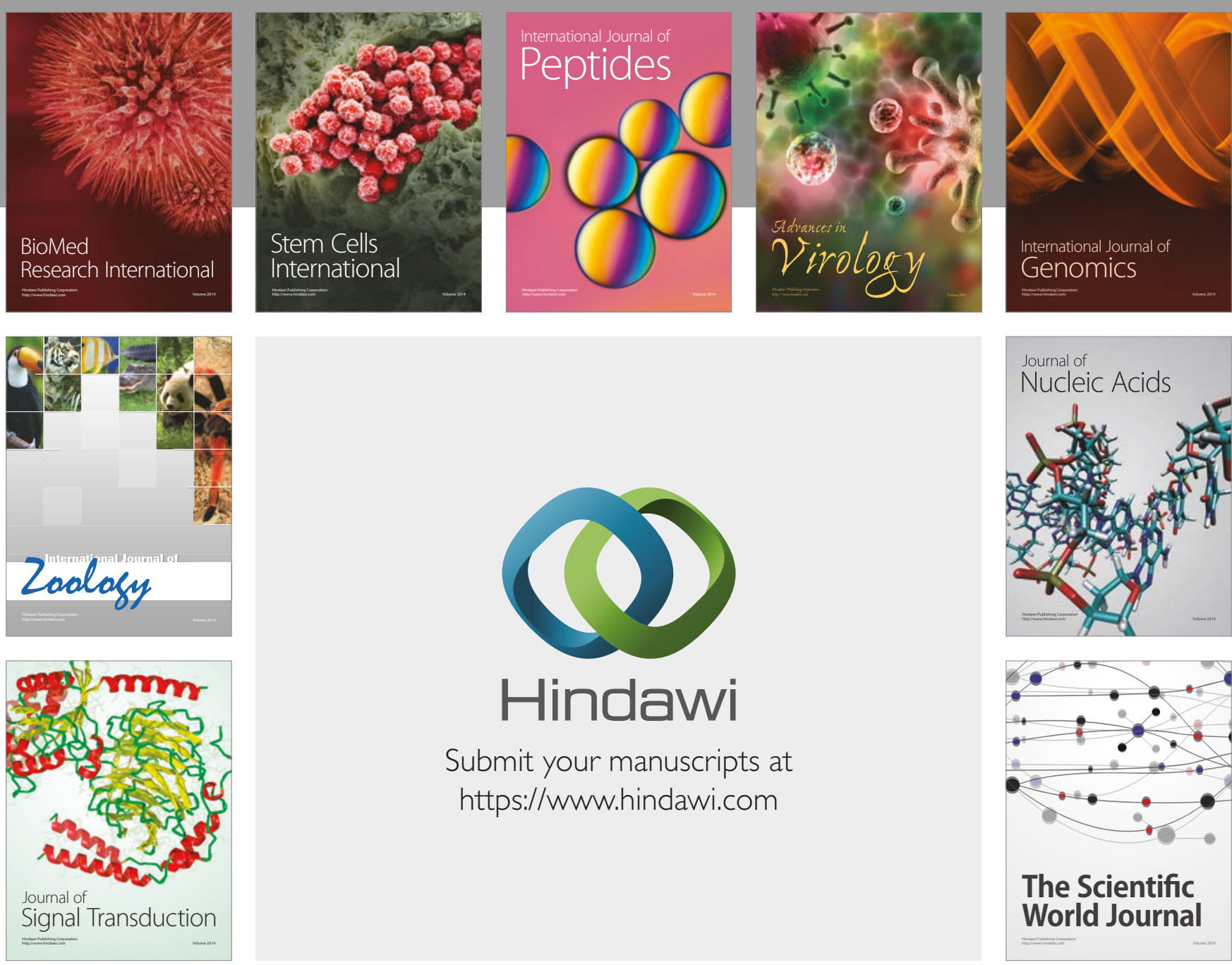

Submit your manuscripts at

https://www.hindawi.com
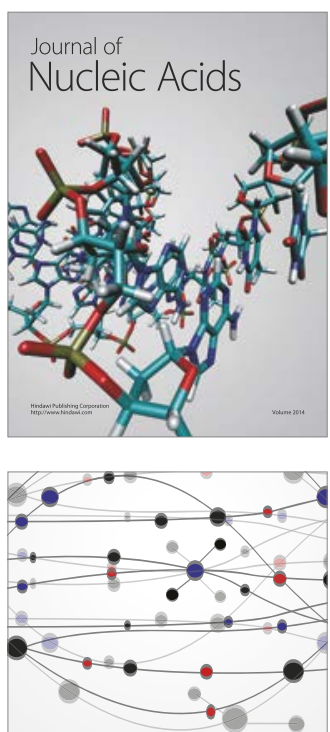

The Scientific World Journal

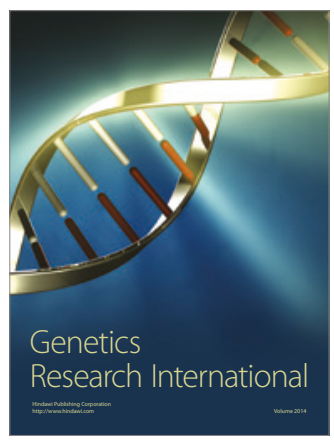

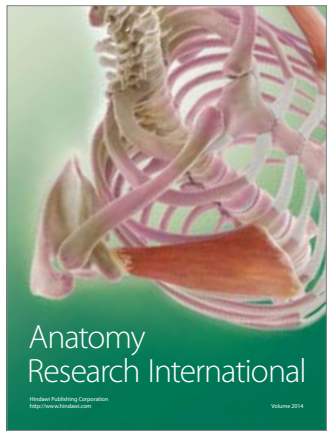

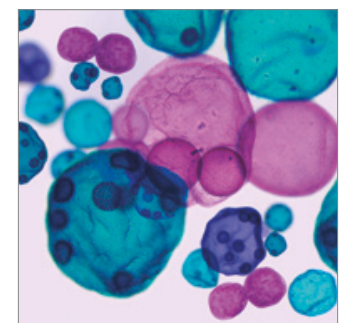

International Journal of Microbiology
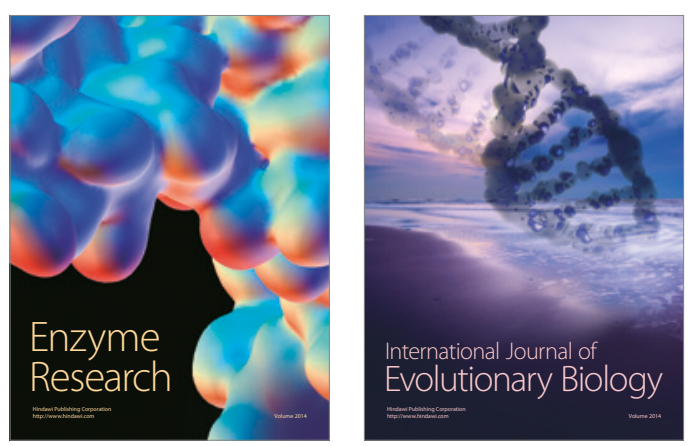
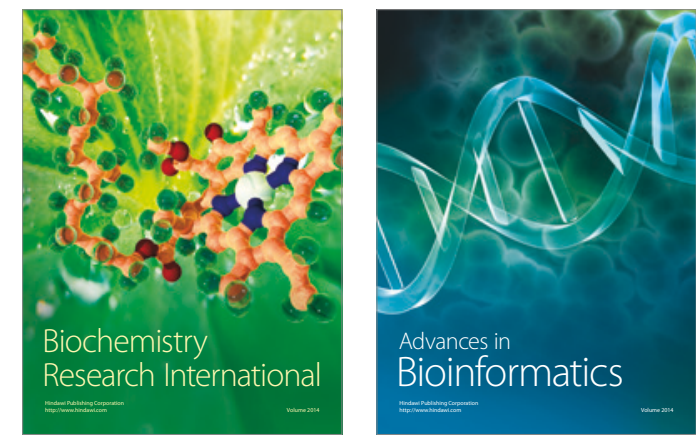

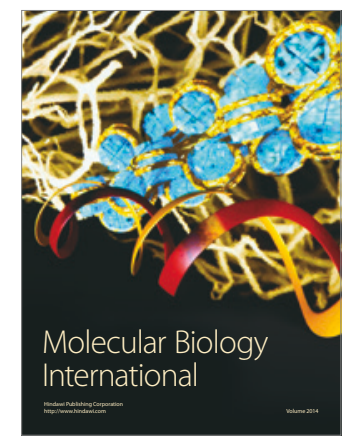

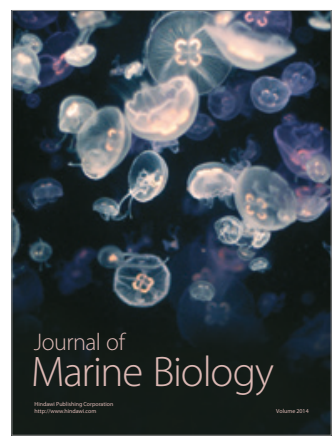

